\title{
Observed frequency and challenges of variant reclassification in a hereditary cancer clinic
}

\author{
Sarah Macklin, $\mathrm{MMSc}^{1}$, Nisha Durand, $\mathrm{PhD}^{2}$, Paldeep Atwal, MB, ChB ${ }^{1,3}$ and Stephanie Hines, MD
}

Purpose: Efforts have been made by the American College of Medical Genetics and Genomics and the Association for Molecular Pathology to make variant classification more uniform, but many limitations remain. Reclassification of a variant of uncertain significance (VUS) is expected, but other more certain calls, like pathogenic or benign, can also be reclassified once additional information is gathered. Variant reclassification can create difficult circumstances for both patients and clinicians.

Methods: Retrospective review of all variant reclassifications in genes associated with hereditary cancer syndromes at one clinic between September 2013 and February 2017 was completed. All variant reclassifications were completed and reported by the original testing laboratory.

Results: A total of 1,103 hereditary cancer tests were ordered. Fewer than $5 \%(40 / 1,103)$ of the initial reports were updated during that time period. Most reclassifications (29/40) were downgrades of VUS to likely benign. Only three reclassifications could potentially alter medical management.

Conclusion: The majority of variant reclassifications do not impact medical management. Upgrading a variant call to pathogenic could be important for a patient's care and shows the importance of open communication between laboratories and clinicians. A variant downgrade from pathogenic can be a significant reclassification as well, especially if prophylactic surgery has been completed.

Genet Med advance online publication 7 December 2017

Key Words: hereditary cancer clinic; hereditary cancer syndrome; variant classification; variant of uncertain significance; variant reclassification

\section{INTRODUCTION}

Genetic testing has become increasingly routine, with an increase in laboratories offering testing and a decrease in testing cost opening up accessibility. ${ }^{1}$ More nongenetics specialists, such as primary care providers, oncologists, and gynecologists, are ordering genetic testing for hereditary cancer syndromes; this increase provides an influx of patients trying to integrate genetic test results into their medical management. ${ }^{2,3}$

When a hereditary cancer syndrome is identified, this can provide patients with important information about risk to help guide medical management decisions. For women with a pathogenic variant in BRCA1/2, management can include a variety of options from high-risk surveillance to prophylactic removal of the ovaries and/or breasts. ${ }^{4}$ These recommendations are based on the associated risks for cancer in this population, with recent risk estimates around $69-72 \%$ for breast cancer and $17-44 \%$ for ovarian cancer. ${ }^{5}$ Removal of the ovaries in women with $B R C A 1 / 2$ pathogenic variants reduces cancer risk by around 96\%. ${ }^{6}$ Prophylactic removal of the breasts reduces breast cancer risk by around $90 \%$ in this population. ${ }^{7}$ Other hereditary causes of breast cancer may require increased surveillance and/or risk-reducing mastectomy as well. ${ }^{4}$ Similarly, individuals with Lynch syndrome may be offered increased colonoscopy surveillance and/or colectomy. ${ }^{8}$ Although positive test results can help patients make decisions about whether high-risk screening and/or prophylactic surgery is right for them, not all individuals who pursue testing receive a straightforward result.

Genetic variants can be classified into one of five categories based on relevant, available data: pathogenic, likely pathogenic, variant of uncertain significance (VUS), likely benign, and benign. The word likely in this context roughly equals a greater than $90 \%$ certainty that a specific variant is pathogenic or benign. ${ }^{9}$ Variants of uncertain significance are genetic variants with an unknown impact on health, leaving both patients and providers uncertain about their relevance. At least one VUS is reported about one-third of the time when a 25 -gene cancer susceptibility panel is ordered. ${ }^{10}$ The goal is to reclassify an inconclusive result as benign or pathogenic as more is understood about that specific genetic change. However, research has shown that patients do not always fully comprehend the uncertainty of a VUS. ${ }^{11,12}$ In one study, nearly $80 \%$ of patients thought that having a VUS in BRCA1/2 at least slightly increased their risk for cancer. ${ }^{11}$ Similar results were found in individuals carrying a VUS in a Lynch syndrome-associated gene. ${ }^{12}$ These results are clearly not always well communicated to patients.

${ }^{1}$ Department of Clinical Genomics, Mayo Clinic, Jacksonville, Florida, USA; ${ }^{2}$ Laboratory Medicine and Pathology, Mayo Clinic, Jacksonville, Florida, USA; ${ }^{3}$ Center for Individualized Medicine, Mayo Clinic, Jacksonville, Florida, USA; ${ }^{4}$ Department of Medicine, Division of Diagnostic \& Consultative Medicine, Mayo Clinic, Jacksonville, Florida, USA. Correspondence: Sarah Macklin (macklin.sarah@mayo.edu)

Submitted 18 October 2017; accepted 18 October 2017; advance online publication 7 December 2017. doi:10.1038/gim.2017.207 
Additional challenges exist when utilizing genetic test results in clinical practice. Although patients rely on the accuracy of their genetic results to make potentially lifealtering medical decisions, not all laboratories classify genetic variants identically. Guidelines have been created by the American College of Medical Genetics and Genomics and the Association for Molecular Pathology (ACMG-AMP) to create continuity between how laboratories weigh available data. ${ }^{9}$ Initial reports show there is still work to be completed. Nine laboratories were asked to classify variants based on the guidelines, and there was complete agreement on calls around one-third of the time (33/99). ${ }^{13}$ This was not statistically different than when the laboratories used their own criteria for classification. ${ }^{13}$ When the laboratories discussed their discrepancies and used the ACMG-AMP guideline, a consensus of $71 \%$ on variant calls (70/99) was achieved. ${ }^{13}$ This is a significant improvement, but total cohesion is still difficult to achieve. Full discussion between laboratories for each call is not feasible for many reasons.

Another challenge occurs when a result initially reported as benign or pathogenic is reclassified based on the availability of new information. Reclassifications may have significant ramifications if medical management decisions have been made based on the previous interpretation of results. Genetic testing is increasingly utilized in management earlier after an initial diagnosis, which could increase risk for variant reclassification following an intervention. ${ }^{1}$ For one clinic, only $4 \%$ of breast cancer patients had genetic testing completed during neoadjuvant chemotherapy in 2006; this number increased to $25 \%$ by $2014(p=0.002) .{ }^{1}$ About half of women found to carry a pathogenic BRCA1/2 variant who choose prophylactic surgery have surgery completed within half a year after diagnosis. ${ }^{14}$ Around $75 \%$ (225/305) of $B R C A 1 / 2$ positive women elected to have a risk-reducing bilateral salpingo-oophorectomy with median time from diagnosis being 6 months (3-11 months). ${ }^{14} \mathrm{~A}$ little less than half $(110 / 250)$ performed risk-reducing bilateral mastectomy with a median time of 6 months as well. ${ }^{14}$

Unfortunately, it can take years before enough data is gathered to reclassify a variant. Around one quarter (5/22) of the women with a VUS at one clinic followed for 8 years or longer still had not received a definitive result. ${ }^{15}$ At another clinic, $56 \%$ of women with a VUS $(N=69)$ received a reclassification after a median of 39 months. ${ }^{16}$ Little is known about the likelihood of a likely pathogenic/pathogenic variant being downgraded or a likely benign/benign variant being upgraded. The purpose of this study was to better understand the likelihood of reclassification of genetic variants during a 4year time period. It was hypothesized that most reclassifications would not affect medical management and that many reclassifications would have occurred following the 2015 ACMG-AMP guidelines.

\section{MATERIALS AND METHODS}

A retrospective review was performed on all genetic test results that were received on patients who presented for a possible hereditary cancer syndrome and completed genetic testing through one US-based commercial genetic testing laboratory. All patients were evaluated at the Mayo Clinic in Florida between September 2013 and February 2017 and received genetic counseling from a genetic counselor and/or physician certified in medical genetics. All initial test results and any updated reclassification reports were reviewed. All reported reclassifications were made by the original testing lab.

\section{RESULTS}

A total of 1,119 gene panels were ordered to test patients for hereditary cancer syndromes at the Mayo Clinic in Jacksonville, Florida between September 2013 and February 2017. Sixteen reports were not included in analysis as a copy of the original report could not be located in the electronic medical record. No reclassifications had been reported in any of these 16 reports. A total of 1,103 reports were included in analysis (Table 1). The majority of the panels analyzed multiple genes related to breast and/or ovarian cancer. Around 10\% $(111 / 1,103)$ discovered a pathogenic or likely pathogenic variant. A total of 266 VUS were originally reported in 226 individuals (Table 1). Ten (4.4\%) of those with a VUS also had a pathogenic variant identified. The majority of the tests did not identify any (likely) pathogenic variants or VUS (776/1,103 (70.4\%)).

Of the 1,103 genetic test results obtained, 3.6\% $(40 / 1,103)$ have been updated since the original report. Efforts were made to share the updated test reports with each patient. The clinic was able to make confirmed contact regarding 35 of the 40 reclassifications. Three patients were unable to be reached via telephone or electronic health system portal, but a copy of the updated report and an explanation was mailed to the address on file via certified mail. Two other participants died before the reclassification occurred.

Among the reclassifications, the majority were initially classified as VUS, but overall, only a small portion of the VUS were reclassified during this time period $(11.3 \%(30 / 266))$. Around 75\% (29/40) of the reclassified variants were initially

Table 1 Distribution of testing ordered and VUS

\begin{tabular}{lcc} 
Test & $\begin{array}{c}\text { \# Ordered } \\
\text { (\% of total) }\end{array}$ & $\begin{array}{c}\text { No. of VUS } \\
\text { (\% of total VUS) }\end{array}$ \\
\hline $\begin{array}{l}\text { Breast and/or ovarian } \\
\text { cancer panel }\end{array}$ & $552(50.0 \%)$ & $184(69.2 \%)$ \\
\hline$B R C A 1 / 2$ only & $180(16.3 \%)$ & $11(4.1 \%)$ \\
\hline Custom panel & $135(12.2 \%)$ & $27(10.2 \%)$ \\
\hline Multicancer panel & $64(5.8 \%)$ & $20(7.5 \%)$ \\
\hline Colorectal cancer panel & $49(4.4 \%)$ & $10(3.8 \%)$ \\
\hline Targeted variant testing & $46(4.2 \%)$ & $1(0.4 \%)$ \\
\hline Pancreatic cancer panel & $31(2.8 \%)$ & $9(3.4 \%)$ \\
\hline Single-gene testing & $26(2.4 \%)$ & $2(0.8 \%)$ \\
\hline Endometrial cancer & $20(1.8 \%)$ & $2(0.8 \%)$ \\
panel & & \\
\hline
\end{tabular}

VUS, variants of uncertain significance. 
classified as a VUS and now have been reclassified as likely benign variants (Figure 1). About 7\% (3/40) of the reclassifications were likely benign variants downgraded to benign variants, and three likely pathogenic variants were reclassified as pathogenic. All of the original likely benign variants were reported before these calls were removed from this laboratory's reports.

Overall, three variants received reclassifications that could affect medical management, including one likely pathogenic variant and one pathogenic variant that have been reclassified as VUS (Table 2). The patient with the BRIP1 variant previously called pathogenic had proceeded with a bilateral salpingo-oophorectomy before reclassification. This surgery was completed in conjunction with another required and unrelated abdominal procedure. It had been discussed with the patient that there was limited information available regarding exact risk for ovarian cancer for carriers of a pathogenic BRIP1 variant and efficacy of risk-reducing oophorectomy was unknown. No prophylactic surgeries were performed on the patient with the BRCA2 variant classified previously as likely pathogenic. One MSH6 VUS was reclassified as likely pathogenic. This individual was contacted and appropriate management recommendations were discussed. One pathogenic variant was downgraded to likely pathogenic and one likely benign variant was upgraded to a VUS. No changes to management were made based on these new calls, but the information was important to share with the two patients.

The majority of the reclassifications (35/40) were made after the ACMG-AMP released guidelines for classifying genetic variants in May 2015. This is likely impacted by the number

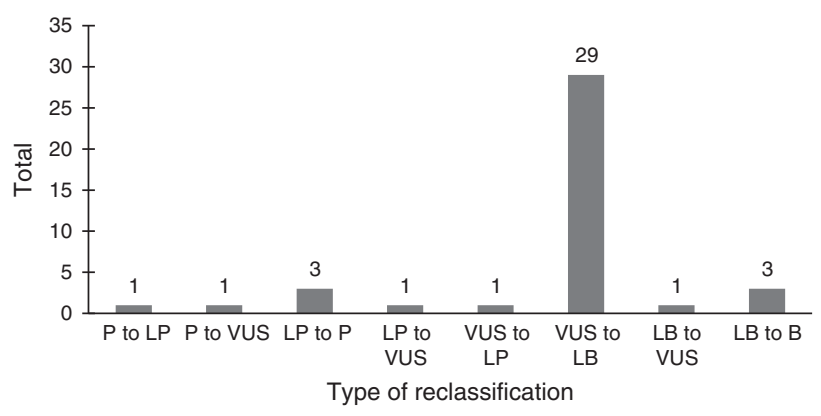

Figure 1 Types of variant reclassifications observed. $B$, benign; LB, likely benign; LP, likely pathogenic; P, pathogenic; VUS, variant of uncertain significance. of follow-up years before and after the guideline publication dates, but 20/35 (57\%) reclassifications made after May 2015 cited review of the variant in light of the 2015 ACMG- AMP guidelines as at least partial reason for reclassification.

\section{DISCUSSION}

The clinic was able to confirm that the majority of the patients were updated regarding their variant's new classification. Not all were able to be reached. Part of the posttest counseling for a patient with a VUS should always include a recommendation that the patient follows up with the ordering provider every 2-3 years to learn about current status of the variant. Follow-up from the patient could create an opportunity for disclosure that a change in address or phone number could deny. All results that had an impact on management were communicated over the phone and/or in person and not through the online portal or letter.

Overall, most reclassifications were downgrades of VUS. This type of reclassification should not have a significant impact on the patient's care as it is not typically recommended that medical management decisions be based on a VUS. For patients who are diagnosed with a VUS, medical management should be based on an individual risk that is determined by the patient's personal and family history. About $15 \%$ of the reclassifications were variants confirmed to be pathogenic or benign from likely pathogenic or likely benign. These reclassifications should not significantly impact patient care as "likely" calls are often treated similarly to confirmed calls.

Only 3 of the 40 reclassifications (7.5\%) could potentially affect patient care at this time: the downgrade of a likely pathogenic variant and a pathogenic variant to VUS and the upgrade of a VUS to likely pathogenic. Upgrading a VUS to a likely pathogenic variant would warrant a reassessment of the patient's individual cancer risks and management strategies. Patient management would now take into account the risks and risk-mitigation strategies associated with the specific gene affected. However, the reclassification of a pathogenic or likely pathogenic variant to a VUS, while rare $(0.27 \%$ of all initially reported test results), would also lead to a substantial reassessment of cancer risk and subsequent alteration of medical management that could be problematic in patients who have already undertaken invasive and potentially lifealtering prophylactic surgeries in response to the initial result and corresponding risk. The significance of this type of reclassification also may affect the provider-patient

Table 2 Rationale stated for variant reclassification

\begin{tabular}{llll} 
Gene & Original call & Updated call & Reclassification rationale \\
\hline BRCA1 & Likely benign & Variant of uncertain significance & New classification was more consistent with the 2015 ACMG guidelines \\
\hline$B R C A 2$ & Likely pathogenic & Variant of uncertain significance & New functional data was discordant with original interpretation \\
BRIP1 & Pathogenic & Variant of uncertain significance & Review of internal data collected \\
MSH6 & Variant of uncertain significance & Likely pathogenic & Review of internal data and reevaluation of relevant literature \\
\hline
\end{tabular}

ACMG, American College of Medical Genetics and Genomics. 
relationship as providers attempt to inform patients of the reclassification, its attendant effect on the initial risk assessment, and management strategies.

The downgrading of variants from pathogenic to likely pathogenic or upgrading of a likely benign variant to a VUS would not be expected to affect medical management but may still elicit concern about the variant classification process and the evidence upon which it is based.

It is important for laboratories to continue to evaluate the variant calls as future research will continue to develop our understanding of genes and their associated conditions. Sharing of data and classifications between laboratories, such as deposition of variants into ClinVar, can increase knowledge on how specific genetic variants impact health.

Clinicians, researchers, and laboratory directors frequently use online human variant databases to better understand genetic variants. These databases provide a platform for the critical data sharing needed to sort through the vast information provided through next-generation sequencing. ${ }^{17}$ The National Society of Genetic Counselors has a position statement that supports responsible participation in these types of databases. ${ }^{18}$ Patients can often benefit from these variant databases as laboratory directors and clinicians can view a particular variant with the aggregate data of multiple laboratories. ${ }^{17}$ This can decrease the time needed to classify a variant, which can be important when life-changing medical management decisions are based on genetic test results.

Due to the magnitude of impact that certain genetic test results may bring, laboratory directors may feel a responsibility to not "overcall" variants. Legal questions could arise if a $B R C A 1$ variant was downgraded to benign after previously being called pathogenic. Women may have performed prophylactic surgery based on this result. It is unclear who, if any group, is responsible for this event or if it is a natural risk of medicine in general. For these reasons, it can be in the best interest of the laboratory and patient to confirm that the laboratory has enough relevant data before they classify a variant as pathogenic.

Williams $v$. Athena is a pending lawsuit that examines the legal responsibility of a genetic testing laboratory to provide accurate variant calls in a timely manner. ${ }^{17}$ The plaintiff argues in the case that Athena was negligent in their interpretation of a SNC1A variant. They state that this negligence led to a wrongful death. While evidence of the defendant and the outcome of this case are unknown, this lawsuit prompts conversation about the legal and ethical responsibilities of laboratories, variant databases, and clinicians.

The genetics community has made progress to make variant calls more uniform, but further development is required. BRCA1/2 variant classification discordance between one commercial genetic testing company and a free, online human variant database was studied. ${ }^{19}$ Of 4,250 BRCA1/2 variants, $73.2 \%$ of calls were in complete agreement. About $15 \%$ of classifications were in disagreement. Most of these discordant calls were classified as VUS by the public variant database and either benign or pathogenic by the one commercial laboratory. This discordance illustrates what can be gained by laboratories working together and sharing data. Genetic conditions can vary greatly from one another both molecularly and by phenotype. Valuable time is lost in the care of patients if all testing companies are studying variants independently.

A limitation of this study is that all results and reclassifications were completed at one genetic testing laboratory. Another commercial testing company could have higher or lower numbers of reclassifications. Also, limited follow-up time was available for many results. It is unclear what the reclassification rate may look like 5 or 10 years posttesting. This is an area that deserves further study.

\section{Conclusion}

Many prophylactic surgeries take place within 2 years after someone has been diagnosed with a hereditary cancer syndrome, and the majority of women with pathogenic $B R C A 1 / 2$ variants elect for a risk-reducing bilateral salpingooophorectomy. ${ }^{1,14,16}$ Many other genetic test results are accompanied by significant medical management recommendations as well. Reclassification of variants thought to be likely pathogenic or pathogenic occurs infrequently, but in some of these cases, significant medical management decisions may have already been made. Open communication between laboratories and clinicians may help to provide timely and accurate results to patients. Gene- and disease-specific variant classification guidelines could also help to address some of the limitations of the ACMG-AMP guidelines. ${ }^{20}$ Further investigation into the legal and ethical responsibilities of all parties is required.

\section{DISCLOSURE}

The authors declare no conflict of interest.

\section{REFERENCES}

1. Moreno L. Germline BRCA testing is moving from cancer risk assessment to a predictive biomarker for targeting cancer therapeutics. Clin Trans/ Oncol 2016;18:981-987.

2. Keating NL, Stoeckert KA, Regan MM, DiGianni L, Garber JE. Physicians' experiences with BRCA $1 / 2$ testing in community settings. J Clin Oncol 2008;26:5789-5796.

3. Shields $A E$, Burke W, Levy DE. Differential use of available genetic tests among primary care physicians in the U.S.: results of a national survey. Genet Med 2008;10:404-414.

4. Daly MB, Pilarski R, Berry M, et al. Genetic/Familial High-Risk Assessment: Breast and Ovarian. Version 2.2017. NCCN Clinical Practice Guidelines in Oncology 2016. https://www.nccn.org/professionals/physician_gls/pdf/ genetics_screening.pdf. Accessed 8 August 2017.

5. Kuchenbaecker KB, Hopper JL, Barnes DR, et al. Risks of breast, ovarian, and contralateral breast cancer for BRCA1 and BRCA2 mutation carriers. JAMA 2017;317:2402-2416.

6. Olopade OI, Artiolo G. Efficacy of risk- reducing salpingo-oophorectomy in women with BRCA1 and BRCA2 mutations. Breast J 2004;10(suppl 1): S5-9.

7. Rebbeck TR, Friebel T, Lynch HT, et al. Bilateral prophylactic mastectomy reduces breast cancer risk in BRCA1 and BRCA2 mutation carriers: the PROSE study group. J Clin Oncol 2004;22:1055-1062.

8. Provenzale D, Gupta S, Ahnen D, et al. Genetic/Familial High-Risk Assessment: Colorectal. Version 2.2017. NCCN Clinical Practice Guidelines in Oncology 2017. https://www.nccn.org/professionals/physician gls/pdf/genetics_colon.pdf. Accessed 5 September 2017. 


\section{ORIGINAL RESEARCH ARTICLE}

9. Richards S, Aziz N, Bick D, et al. Standards and guidelines for the interpretation of sequence variants: a joint consensus recommendation of the American College of Medical Genetics and Genomics and the Association for Molecular Pathology. Genet Med 2015;17: 405-424.

10. Tung $\mathrm{N}$, Lin $\mathrm{NU}$, Kidd J, et al. Frequency of germline mutations in 25 cancer susceptibility genes in a sequential series of patients with breast cancer. J Clin Oncol 2016;34:1460-1468.

11. Vos J, Otten W, van Asperen C, Jansen A, Menko F, Tibben A. The counsellees' view of an unclassified variant in BRCA1/2: recall, interpretation, and impact on life. Psychooncology 2008;17:822-830.

12. Solomon I, Harrington E, Hooker G, et al. Lynch syndrome limbo: patient understanding of variants of uncertain significance. I Genet Counsel. 2017;26:866-877.

13. Amendola LM, Jarvik GP, Leo MC, et al. Performance of ACMG-AMP variant- interpretation guidelines among nine laboratories in the clinical sequencing exploratory research consortium. Am J Hum Genet 2016;98: 1067-1076.
14. Garcia C. Risk management options elected by women after testing positive for a BRCA mutation. Gynecol Oncol 2014;132:428-433.

15. Murray ML. Follow up of carriers of BRCA1 and BRCA2 variants of unknown significance: variant reclassification and surgical decisions. Genet Med 2011;13:998-1005.

16. Garcia C. Comparison of risk management strategies between women testing positive for a BRCA variant of unknown significance and women with known BRCA deleterious mutations. Genet Med 2014;16:896-902.

17. Thorogood A, Cook-Deegan R, Knoppers BM. Public variant databases: liability?. Genet Med 2017;19:838-841.

18. National Society of Genetic Counselors. Position Statements: Clinical Data Sharing. http://www.nsgc.org/p/bl/et/blogaid $=330$. Accessed 6 September 2017.

19. Gradishar W, Johnson K, Brown K, Mundt E, Manley S. Clinical variant classification: a comparison of public databases and a commercial testing laboratory. Oncologist 2017;22:797-803.

20. Hoskinson DC, Dubuc AM, Mason-Suares $H$. The current state of clinical interpretation of sequence variants. Curr Opin Genet Dev 2017;42:33-39. 